\title{
IMPLICATIONS OF SPATIAL VARIABILITY CHARACTERIZATION IN DISCRETE PARTICLE MODELS
}

\section{JAN PODROUŽEK ${ }^{\dagger *}$, JAN VOREL ${ }^{*}$, IOANNIS BOUMAKIS ${ }^{*}$, GIANLUCA CUSATIS ${ }^{\dagger \dagger}$, ROMAN WENDNER*} \author{
University of Natural Resources and Life Sciences, \\ Vienna, Austria \\ e-mail: roman.wendner@boku.ac.at \\ ${ }^{\dagger}$ Brno University of Technology \\ Czech Republic \\ e-mail: podrouzekj@fce.vutbr.cz \\ ${ }^{\dagger}$ Northwestern University \\ Illinois, United States \\ e-mail: g-cusatis@northwestern.edu
}

Christian Doppler Laboratory LiCRoFast, Institute of Structural Engineering,

Key words: Random fields, Composite materials, Monte Carlo, Sample selection

\begin{abstract}
Characterization of the inherent spatial variability of the mechanical properties of advanced composite materials as well as of standard concretes is among the primary concerns of the presented paper. In the context of discrete particle models the random field concept is frequently adopted in order to account for the fluctuations of material characteristics on scales independent of the geometrical characterization of the mesostructure as represented by particular particle configurations mimicking the aggregate placement. The specific goal of this paper is to introduce spatial variability into structural reliability by providing a sample reduction strategy for the discrete framework. Presented simulations of a classical experiment utilize the well-established Lattice Discrete Particle Model (LDPM) and demonstrate the general potential of the proposed strategy towards current state-of-the-art in stochastic mechanics and some relating open problems.
\end{abstract}

\section{INTRODUCTION}

An increased demand over the last few decades in developing procedures for statistical estimates of structural response has been driven by the growing number of deteriorating structures, many of which are still in use due to socio-economic constraints, and by the increasingly available computational resources $[1,2]$. This has prompted research into Monte Carlo (MC) based small-sample simulation methods [3,4], where, despite the capacity of current computers, among the essential barriers preventing practical utilization still remains the necessity of adopting an effective sampling strategy that would reduce dramatically the number of required realizations while maintaining accurate estimates of the response characteristics (low-probability largeconsequence events) [5].

\subsection{Small sample simulations for spatial variability}

In the context of engineering risk analysis, the structural models typically do not have a 
closed-form solution and a single evaluation is already computationally demanding. In some cases, the sensitivity of response to individual input parameters, such as material, technological or environmental characteristics can be estimated by the deterministic study of the problem (identify unfavourable conditions) and a sampling strategy can be effectively formulated. This is routinely done e.g. in structural static analysis with randomized scalar input variables, such as material parameters $[6,7]$. With some exceptions, such approach yields reasonable results in terms of accuracy and computational cost, unless due to high number of random input variables, extremely complex (or ill-defined) structural model, or simply due to solver noise, the particular inputoutput dependency becomes non-monotonous, as for example in the case of a highly prestressed bridge, where more favourable redistribution due to weakened elasticity modulus can lead to an increased load bearing capacity [8].

As for spatial variability in structural safety, the importance of which is indicated by a number of publications, the situation in terms of available sampling strategy is different. Unlike in the previous case of scalar random input variables, the sensitivity of individual input patterns (random fields) cannot be obtained by a deterministic study of the problem and the likelihoods cannot be easily assigned to individual realizations of such patterns due to obvious reasons [9], unlike in the case of e.g. concrete characteristics, whose statistical description (correlated scalars) can be based on experimental campaigns [10], where the effect of inherent spatial variability is already reflected in the higher moments of probability distributions of e.g. tensile strength, compressive strength, modulus of elasticity, specific fracture energy etc. Here it should be noted that in most cases the spatial heterogeneity at arbitrary scale (meso, micro and macro) is irrelevant for the assessment of engineering structures made from basic construction materials, such as concrete, steel or wood. However, it becomes highly relevant to currently designed composites [11], structural details such as those of anchoring technology [12] or geotechnical assessment [13]. Similarly, the implicit treatment of spatial variability is important to the reliability based code calibration $[14,15]$, design of experiments [16] and verification of various hypotheses or limit theorems, such as fracture behaviour or size effect [17].

\subsection{Combined phenomena}

Concrete is often approximated as statistically homogenous at macroscopic scale, yet its macroscopic behaviour demonstrates strain softening, quasi-brittleness and size effect [18]. The appearance and evolution of cracking due to mechanical loading and environmental exposure can be as well partially attributed to material heterogeneity of concrete. In order to estimate a multi-decade performance of concrete structures, not only the material heterogeneity have to be taken in to account, but also the spatially variable boundary conditions (orientation and location of the exposed surfaces) and stochastic processes (e.g. weather conditions) involved [19]. Among the typical phenomena of deterioration are salt injury, freezing and thawing damage, carbonation and chemical attack.

Understanding the combined effects of environmental exposure and concrete deterioration is still subjected to research as an open problem. Similarly, characterization of long-term mechanical phenomena, such as creep and shrinkage, and coupling with hygrothermal diffusion/transfer processes and the effect of damage on diffusivity properties is considered an open question and subjected to research.

Physical experiments are clearly limited when multi-decade characterization is to be considered. Accelerated tests may reduce the time requirements significantly, but are clearly limited when high number of experimental setup combinations have to be evaluated or if repeated statistical experiments are planned. Not surprisingly, numerical simulations are more frequently used as a primary tool to approach or solve some of the most challenging open problems of the current time. 


\subsection{LDPM}

A well-established model naturally accounting for material heterogeneity, the Lattice Discrete Particle Model [20-22], has been extensively calibrated and validated and it has shown superior capabilities in reproducing and predicting concrete behaviour. It simulates the mesostructure of concrete by a threedimensional (3D) assemblage of particles (fig. 1) that are generated randomly according to a given grain size distribution. Delaunay tetrahedralization and 3D domain tessellation are used here to generate a system of cells interacting through triangular facets. Displacements and rotations of such adjacent particles form the discrete compatibility equations in terms of rigid body kinematics. At each cell facet the mesoscale constitutive law is formulated such that it simulates cohesive fracture, compaction due to pose collapse, frictional slip and rate effect. For each single particle an equilibrium equations are finally formulated. An extended version of LDPM is currently developed and simulates various coupled deterioration mechanisms, such as Alkali-Silica reaction (ASR) [22,23].

\section{IDENTIFICATION STRATEGY}

The amount of variability concrete specimens and structural systems may exhibit due to aggregate orientation, size, shape and ratio is well known. Magnitude of the influence of these microstructural differences on the composite's properties will result in a scatter of response characteristics, such as load displacement curves or crack propagation.

In order to reduce the prohibitive computational cost of repeated highdimensional LDPM stochastic simulations in an attempt to capture the scattering characteristics, the following identification strategy is proposed.

This was originally formulated by Podroužek et al. [9,19] and presented in the context of seismic risk analysis, where the stochastic process represented ground motion events.

The main objective here is to identify critical realizations $r_{c r i t}$ of LDPM (or/and additional stochastic process) from a finite target sample set $\mathbf{S}$ (of LDPM realizations $r_{i}$ ) under defined critical response criteria, such as resistance, displacement, etc. In the following text the symbol $\rightarrow$ designates higher order mapping function, e.g. $x_{0} \rightarrow f\left(x_{0}\right)$.

\subsection{Proposed identification strategy steps}

s1) Construct a training subset $s$ randomly sampled from $S$ having the length $m<<n$.

$$
\boldsymbol{s} \in\left\langle r_{1}, r_{2}, \ldots, r_{m}\right\rangle
$$

s2) Solve the training subset $s$ :

$$
\left\langle F\left(r_{1}\right), F\left(r_{2}\right), \ldots, F\left(r_{m}\right)\right\rangle \rightarrow \boldsymbol{s}_{\boldsymbol{F}} \in\left\langle y_{1}, y_{2}, \ldots y_{m}\right\rangle
$$

s3) Create ranked minimum and maximum sets $\boldsymbol{s}_{F, \min }$ and $\boldsymbol{s}_{F, \text { max }}$ :

$\boldsymbol{s}_{\boldsymbol{F}, \boldsymbol{m i n}}=\langle 1, \ldots, p\rangle^{\text {th }}$ smallest elements in $\boldsymbol{s}_{\boldsymbol{F}}$

$\boldsymbol{s}_{\boldsymbol{F}, \boldsymbol{m a x}}=\langle 1, \ldots, p\rangle^{t h}$ largest elements in $\boldsymbol{s}_{\boldsymbol{F}}$

s4) Interpolate and reduce (IR transform) the $\boldsymbol{r}_{\boldsymbol{i}}$ 's state variables corresponding to $\boldsymbol{s}_{\boldsymbol{F}, \boldsymbol{m i n}}$ and $\boldsymbol{s}_{\boldsymbol{F}, \boldsymbol{m a x}}$ from the original irregular grid into a joint regular grid

$$
\begin{gathered}
I R\left(\boldsymbol{r}_{\boldsymbol{S}_{\boldsymbol{F}, \min }}\right), \operatorname{IR}\left(\boldsymbol{r}_{\boldsymbol{S}_{\boldsymbol{F}, \boldsymbol{m a x}}}\right) \rightarrow \\
\left\langle\boldsymbol{G}_{\boldsymbol{m i n}, \mathbf{1}}, \ldots, \boldsymbol{G}_{\boldsymbol{m i n}, \boldsymbol{p}}\right\rangle,\left\langle\boldsymbol{G}_{\boldsymbol{m a x}, \mathbf{1}}, \ldots, \boldsymbol{G}_{\boldsymbol{m a x}, \boldsymbol{p}}\right\rangle
\end{gathered}
$$

s5) Formulate a finite set of rules $\boldsymbol{R}$ such that consistently maps the s4) products to the corresponding few important (i.e. maximal or critical) response criteria. Note that a one-toone correspondence is likely unfeasible and the search domain can be effectively narrowed by ignoring the state variables $\left(c_{x, y, z}\right)$ with constant or random behaviour. A simple specific form of $\boldsymbol{R}$ can be attained by calculating the 3- 
Dimensional correlation pattern $\boldsymbol{P}$ using the $\boldsymbol{G}_{\min }$ and $\boldsymbol{G}_{\max }$ :

$$
\begin{aligned}
& \boldsymbol{P} \\
& =\mid \sum_{i=1}^{p} \sum_{x_{\min }}^{x_{\max }} \sum_{y_{\min }}^{y_{\max }} \sum_{z_{\min }}^{z_{\max }} \frac{c_{x, y, z}^{(\min , i)}}{p} \\
& -\sum_{i=1}^{x_{\max }} \sum_{x_{\min }}^{y_{\max }} \sum_{y_{\min }}^{z_{\max }} \sum_{z_{\min }}^{c_{x, y, z}^{(\max , i)}}
\end{aligned}
$$

From the above it is clear that $\boldsymbol{P}$ has the same dimension as the regular grid $\boldsymbol{G}$ and that the higher values $\boldsymbol{P}$ coefficients hold, the more relevant this location became for the classification of the original set $\boldsymbol{S}$. For a sample correlation pattern please refer to fig. 3 . In the following text a concrete form of $\boldsymbol{R}$, i.e. $\boldsymbol{P}$, will be used to help the reproducibility of the proposed strategy.

S6) Quantification of important Features $\boldsymbol{Q F _ { i }}$ on the original set $S$ using the summation of $j^{\text {th }}$ largest element in $\mathbf{P}$ (denoted as $P_{l e}(j)$ ) multiplied by $\boldsymbol{G}_{i}$ element at corresponding position of $j^{\text {th }}$ largest element in $\boldsymbol{P}$ in terms of $x, y, z$ indices, denoted as $G_{c p}(i, j)$ projection.

$$
\boldsymbol{S} \stackrel{I F\left(r_{i}\right)}{\longrightarrow} \boldsymbol{G}_{\boldsymbol{S}}
$$

$$
\begin{aligned}
& \boldsymbol{Q F}_{\boldsymbol{S}_{f}} \\
& =\left\langle\sum_{j=1} P_{l e}(j) \times G_{c p}(1, j), \sum_{j=1}^{f} P_{l e}(j)\right. \\
& \left.\times G_{c p}(2, j), \ldots, \sum_{j=1}^{f} P_{l e}(j) \times G_{c p}(n, j)\right\rangle
\end{aligned}
$$

s7) Classification of $\boldsymbol{S}$ according to the highest values of $\boldsymbol{Q \boldsymbol { F } _ { s }}$ with respect to the response quantity $y_{i .}$. Both values $Q F_{i}$ and $y_{i}$ are correlated now and the identification results are known. The associated likelihoods can be approached as evidence supporting the hypotheses $y_{i} \geq y_{\max }$ :

$$
\operatorname{Probability}\left(y_{i} \geq y_{\max }\right)=\frac{1}{n}
$$

In the broader context one should consider this strategy as a fast MC samples pre-screen to limit the number of necessary LDPM executions. It is assumed that mechanisms behind rules extracted from reasonably small samples are applicable to arbitrarily larger sample. Clearly, whenever using a black-box type of approach, there is a risk of extracting mechanisms that apply only to the training sample if its sample size is too small or in cases of "statistical bad luck". The determination of minimal size of a training set should be based on a requirement for predictive confidence and quantified by associated performance index.

\section{EXPERIMENT}

In order to demonstrate the specific aspects of LDPM modelling and spatial variability, the classical three point bending test was selected. Dimensions of the specimen are $0.1 \times 0.1 \mathrm{x}$ $0.4 \mathrm{~m}$. This volume is approximated by 7608 nodes (fig. 1) and the concrete characterization is briefly described in table 1 .

In terms of peak resistance, which has been used as response criteria for the identification strategy, the 20 realizations resulted in a mean value of $4.27 \mathrm{kN}$ and a coefficient of variation of $4.5 \%$. This scatter (figure 2) can be attributed to the random particle placement only. Each realization run time accounts to approx. 7 hours on one node of the Vienna Scientific Cluster [24].

Table 1. LDPM material characterization.

\begin{tabular}{lcc}
\hline parameter & value & [unit] \\
\hline CementContent & 240. & $\mathrm{~kg} / \mathrm{m} 3$ \\
WaterToCementRatio & 0.83 & - \\
AggregateToCementRatio & 8.83 & - \\
MinAggregate & 0.004 & $\mathrm{~m}$ \\
MaxAggregate & 0.018 & $\mathrm{~m}$ \\
FullerCoefficient & 0.5 & - \\
\hline
\end{tabular}




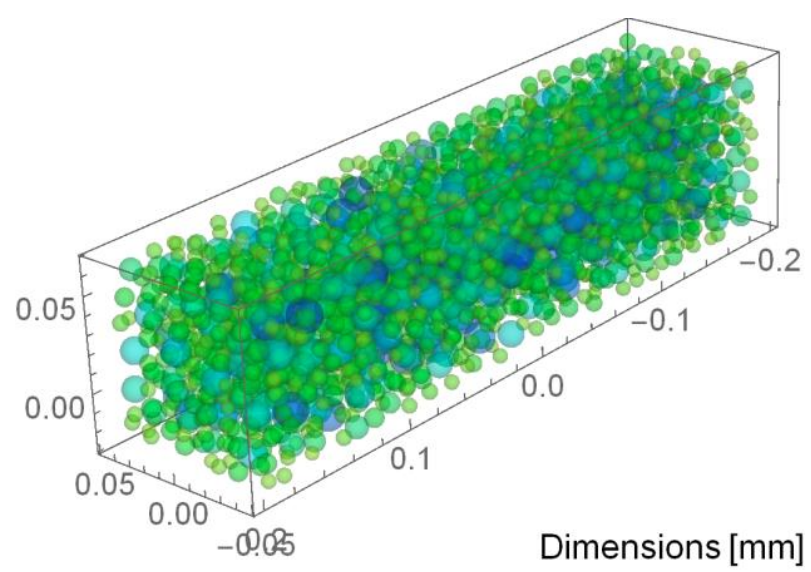

Figure 1: Visualization of a particular LDPM realization of the $3 \mathrm{~PB}$ experiment

\section{RESULTS}

In this paper we consider a lattice discrete particle model of a classical experiment. The inherent material heterogeneity is imposed only via random particle placement and size and leads to a scattered response characteristic, which is depicted at fig. 2. The objective, however, was to introduce the identification strategy to the LDPM framework. The resulting correlation structure (fig. 3, fig. 4) may be further used to identify extreme realizations among arbitrary high number of realizations of the same LDPM experiment (target sample set), or to reproduce statistical characteristics of such set, as e.g. the probability density function.

Given the computational cost of a single execution on a VCS [24], engineering failure probabilities $\left(\sim<10^{-4}\right)$ based on brute-force Monte Carlo are computationally prohibitive, even if scientific grid computing is considered.

In order to overcome this barrier, model reduction schemes, such as homogenization, and/or the proposed sample reduction scheme may be deployed. In some cases, however, homogenization techniques reduce the ability to numerically approach and test some highly theoretical hypotheses, such as various aspects of size effect, distributions or physical interpretation of random field descriptors. Furthermore, maintaining the spatially variable mesoscale detail may in combination with the presented identification strategy lead to important contributions in various areas, where reliability or uncertainty characterization requires profound understanding of spatially variable systems.

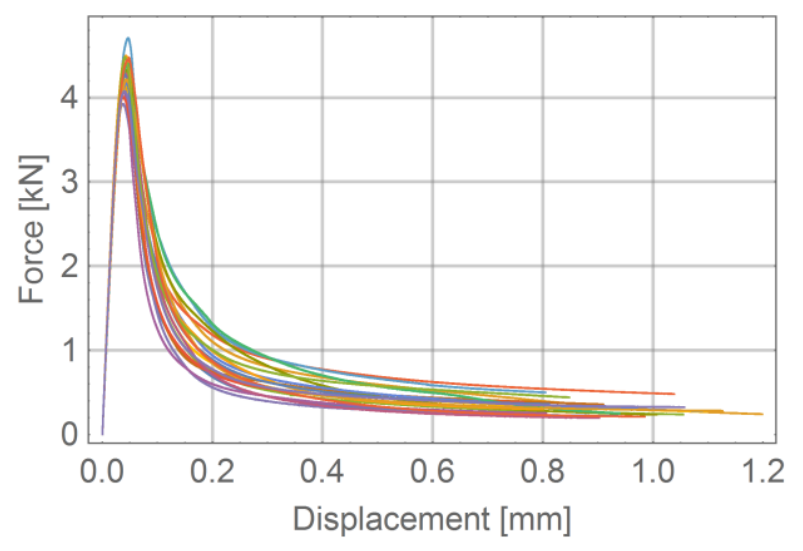

Figure 2: Scattering of 20 realizations of load displacement diagrams.

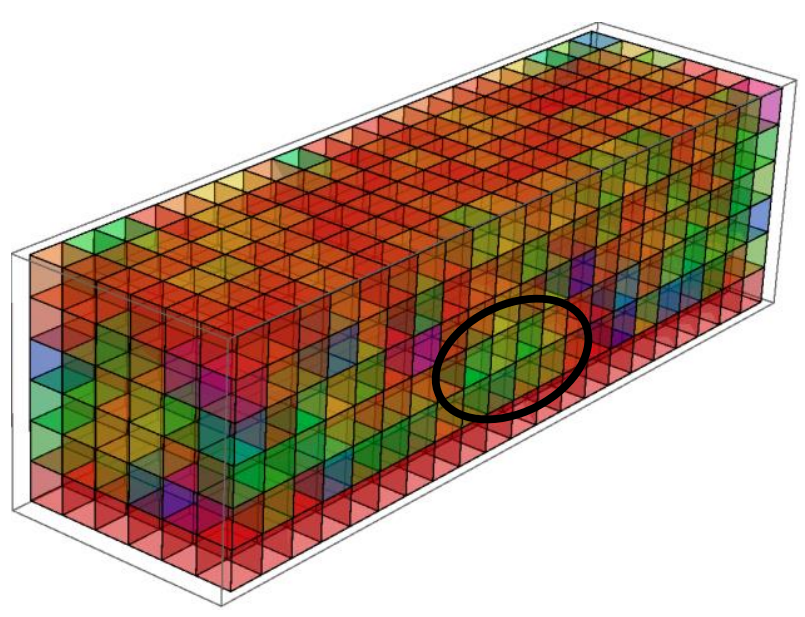

Figure 3: Resulting correlation structure in 3D.

It is out of the scope of this paper to discuss the physical meaning or the interpretation of the resulting correlation structure. It is evident from fig. 3 that no particularly dominant sensitive region has emerged. However, a group of green (more sensitive) cells in in the notched area marked by a black ellipse may suggest the expected sensitivity at the face of the notch. Furthermore, the top and bottom layer appears to be insensitive, which may relate to the actual mechanics of three point bending tests. Despite the triviality of the demonstrated example, it is the authors belief that the proposed strategy 
may provide surprising and unexpected evidence in more complex real-life problems.

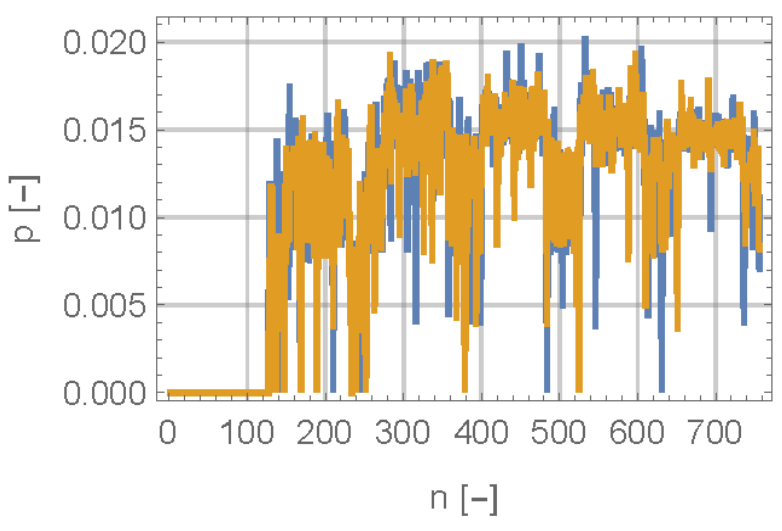

Figure 4: Flatten out correlation structure from fig. 3, where $n$ correlation values $\mathrm{p}$ are plotted in 1D

(blue/yellow line represents maximum/minimum sets).

\section{CONCLUSIONS}

The implications of spatial variability characterization in discrete particle models were discussed and particular aspects presented on a classical three point bending experiment. The predictive stochastic framework for the lattice discrete particle models have to account for multiple sources of uncertainty and heterogeneity. This quickly becomes computationally prohibitive as engineering failure probabilities and Monte Carlo simulations are introduced. The random particle placement can be effectively combined with a random field model to account for spatial variability of material parameters and construction or diffusion/transport processes.

The authors have proposed an identification strategy for correlated random processes and LDPM models, realizations of which may be further classified by the resulting correlation structure. In this way, a significant contribution to the statistical characterization of spatially variable LDPM models can be made, both in terms of first passage probabilities or probability distributions.

\section{ACKNOWLEDGEMENTS}

The financial support by the Austrian Federal Ministry of Economy, Family and Youth and the National Foundation for Research, Technology and Development is gratefully acknowledged. The computational results presented have been achieved in part using the Vienna Scientific Cluster (VSC). The support of the project No. P105/14-10930S provided by the Grant Agency of the Czech Republic is also acknowledged, as well as the project No. LO1408 "AdMaS UP - Advanced Materials, Structures and Technologies", supported by Ministry of Education, Youth and Sports under the "National Sustainability Programme I".

\section{REFERENCES}

[1] Stewart, M.G. 2004 Spatial variability of pitting corrosion and its influence on structural fragility and reliability of RC beams in flexure. Structural Safety, 26, 453-70.

[2] Ellingwood, B.R. 2006 Structural safety special issue: General-purpose software for structural reliability analysis. Structural Safety, 28, 1-2.

[3] Bucher, C. 2009 Computational analysis of randomness in structural mechanics [Internet]. 1st ed. CRC Press, London.

[4] Melchers, R.E. 1999 Structural reliability analysis und prediction. Wiley, Chichester - New York - Brisbane - Toronto.

[5] Ellingwood, B.R. and Wen, Y.-K. 2005 Risk-benefit-based design decisions for low-probability/high consequence earthquake events in Mid-America. Progress in Structural Engineering and Materials, 7, 56-70. http://dx.doi.org/10.1002/pse.191

[6] Bucher, C. 1988 Adaptive Sampling - an iterative fast Monte Carlo procedure. Structural Safety, 5, 119-26. http://dx.doi.org/10.1016/01674730(88)90020-3 
[7] Novák, D., Vořechovský, M. and Rusina, R. 2003 Small-sample probabilistic assessment - FREET software. 9th ICASP Conference, p. 91-6.

[8] Podroužek, J., Strauss, A. and Bergmeister, K. 2014 Robustness-based performance assessment of a prestressed concrete bridge. Structural Concrete, 15, 248-57. http://dx.doi.org/10.1002/suco.201300002

[9] Podroužek, J., Bucher, C. and Deodatis, G. 2014 Identification of critical samples of stochastic processes towards feasible structural reliability applications. Structural Safety, 47, 39-47.

[10] Strauss, A., Zimmermann, T., Lehký, D., Novák, D. and Keršner, Z. 2014 Stochastic fracture-mechanical parameters for the performance-based design of concrete structures. Structural Concrete, 15, 38094.

http://dx.doi.org/10.1002/suco.201300077

[11] Vořechovskỳ, M. and Chudoba, R. 2006 Stochastic modeling of multi-filament yarns: II. Random properties over the length and size effect. International Journal of Solids and Structures, 43, 435-58.

[12]Wendner, R., Vorel, J., Smith, J., Hoover, C.G., Bažant, Z.P. and Cusatis, G. 2014 Characterization of concrete failure behavior: a comprehensive experimental database for the calibration and validation of concrete models. Materials and Structures, 3603-26.

[13] Griffiths, D., Huang, J. and Fenton, G.A. 2009 Influence of spatial variability on slope reliability using 2-D random fields. Journal of Geotechnical and Geoenvironmental Engineering, 135, 1367-78.

[14]fib. 2013 fib Model Code for Concrete Structures 2010. Wilhelm Ernst \& Sohn.

[15]JCSS. 2001 JCSS probabilistic model code; Part I. Basis of design.

[16]Müller, W.G. 2007 Collecting spatial data: optimum design of experiments for random fields. Springer Science \& Business Media.

[17]Bazant, Z.P., Xi, Y. and Reid, S.G. 1991 Statistical size effect in quasi-brittle structures. I. Is Weibull theory applicable? Journal of Engineering Mechanics, 117,
2609-22.

[18] Cusatis, G. and Zhou, X. 2013 High-Order Microplane Theory for Quasi-Brittle Materials with Multiple Characteristic Lengths. Journal of Engineering Mechanics,. http://dx.doi.org/10.1061/(ASCE)EM.194 3-7889.0000747

[19]Podroužek, J., Strauss, A. and Novák, D. 2015 Spatial degradation in reliability assessment of ageing concrete structures. 1st ECCOMAS Thematic Conference on International Conference on Uncertainty Quantification in Computational Sciences and Engineering, Crete, Greece.

[20]Cusatis, G., Pelessone, D. and Mencarelli, A. 2011 Lattice Discrete Particle Model (LDPM) for failure behavior of concrete. I: Theory. Cement and Concrete Composites, 33 , 881-90. http://dx.doi.org/10.1016/j.cemconcomp.2 011.02.011

[21] Schauffert, E. and Cusatis, G. 2012 Lattice Discrete Particle Model for FiberReinforced Concrete. I: Theory. Journal of Engineering Mechanics, 138, 826-33.

[22] Alnaggar, M., Cusatis, G. and Di Luzio, G. 2013 Lattice Discrete Particle Modeling (LDPM) of Alkali Silica Reaction (ASR) deterioration of concrete structures. Cement and Concrete Composites, 41, 4559.

[23]Bažant, Z.P., Zi, G. and Meyer, C. 2000 Fracture mechanics of ASR in concretes with waste glass particles of different sizes. Journal of Engineering Mechanics, 126, 226-32.

[24]2014 Austrian initiative on high performance computing [Internet]. Vienna Sci. Clust. 